\title{
Quality Measures for ETL Processes: From goals to implementation
}

\author{
Vasileios Theodorou ${ }^{1 *}$, Alberto Abelló ${ }^{1}$, Wolfgang Lehner ${ }^{2}$ and Maik Thiele ${ }^{2}$ \\ ${ }^{1}$ Department of Service and Information System Engineering, Universitat Politècnica de Catalunya \\ ${ }^{2}$ Department of Computer Science, Database Technology Group, Technische Universität Dresden
}

\begin{abstract}
SUMMARY
ETL processes play an increasingly important role for the support of modern business operations. These business processes are centred around artifacts with high variability and diverse lifecycles, which correspond to key business entities. The apparent complexity of these activities has been examined through the prism of Business Process Management, mainly focusing on functional requirements and performance optimization. However, the quality dimension has not yet been thoroughly investigated and there is a need for a more human-centric approach to bring them closer to business-users requirements. In this paper we take a first step towards this direction by defining a sound model for ETL process quality characteristics and quantitative measures for each characteristic, based on existing literature. Our model shows dependencies among quality characteristics and can provide the basis for subsequent analysis using Goal Modeling techniques. We showcase the use of Goal Modeling for ETL process design through a use case, where we employ the use of a goal model that includes quantitative components (i.e., indicators) for evaluation and analysis of alternative design decisions.
\end{abstract}

KEY WORDS: ETL; business process; quality measures; Goal Modeling

\section{INTRODUCTION}

Business Intelligence nowadays involves identifying, extracting, and analysing large amount of business data coming from diverse, distributed sources. In order to facilitate decision-makers, complex IT-systems are assigned with the task of integrating heterogeneous data deriving from operational activities and loading of the processed data to data warehouses, in a process known as Extraction Transformation Loading (ETL). This integration requires the execution of real-time, automated, data-centric business processes in a variety of workflow-based tasks. The main challenge is how to turn the integration process design, which has been traditionally predefined for periodic off-line mode execution, into a dynamic, continuous operation that can sufficiently meet end-user needs.

During the past years, there has been considerable research regarding the optimization of ETL flows in terms of functionality and performance [1, 2]. Moreover, in an attempt to manage the complexity of ETL processes on a conceptual level that reflects organizational operations, tools and models from the area of Business Process Management (BPM) have been proposed [3, 4]. These approaches make the crucial step of examining data-centric process models in an outlook that brings them closer to the business core and allows their functional validation and performance evaluation based on user input.

However, the dimension of process quality [5] has not yet been adequately examined in a systematic manner. Unlike other business processes, important quality factors for ETL process

*Correspondence to: vasileios@essi.upc.edu

This is the peer reviewed version of the following article: Theodorou, V., Abelló, A., Lehner, W., Thiele, M. Quality measures for ETL processes: from goals to implementation. "Concurrency and computation. Practice and experience", 1 Octubre 2016, vol. 28, núm. 15, p. 3969-3993, which has been published in final form at http://onlinelibrary.wiley.com/doi/10.1002/cpe.3729/abstract. This article may be used for non-commercial purposes in accordance with Wiley Terms and Conditions for Self-Archiving. 
design are tightly coupled to information quality while depending on the interoperability of distributed engines. Added to that, there is increasing need for process automation in order to become more cost-effective [6] and therefore there needs to be a common ground between analysts and IT that would allow the seamless translation of high level quality concerns to design choices.

The identification of different perspectives of ETL processes - i) data-centric view, ii) software view and iii) business process view - attaches to them an interdisciplinary character and enables the consolidation and reuse of tools and practices from multiple well-established research areas for their analysis. Furthermore, recent advancements in the area of Requirements Engineering offer frameworks targeted to the domain of Business Intelligence that can facilitate ETL process analysis on a business level that is backed by measures and runtime behavior characteristics on the technical level. In addition, the emerging Data Warehousing paradigms of agile design [7] and self-service BI [8] present new opportunities, denoting the necessity of revisiting existing work in the area, in an angle that can promote ETL design automation, while exposing multiple quality dimensions.

In this paper we take a first step towards quality-aware ETL process design automation by defining a set of ETL process quality characteristics and the relationships between them, as well as by providing quantitative measures for each characteristic. For this purpose, we conduct a systematic literature review, extract the relevant quality aspects that have been proposed in literature and adapt them for our case. Subsequently, we produce a model that represents ETL process quality characteristics and the dependencies among them. In addition, we gather from existing literature metrics for monitoring all of these characteristics to quantitatively evaluate ETL processes. Our model can provide the basis for subsequent analysis that will use Goal Modeling techniques [9] to reason and make design decisions for specific use cases, as we showcase through the application of a Goal Model with quantitative components (i.e., indicators) to a running example.

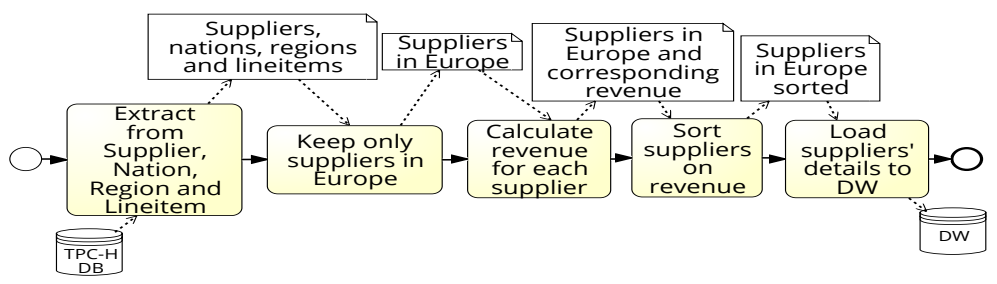

Figure 1.CM_A: A simple conceptual model of the running example ETL

We illustrate how our model works through a running example, based on the TPC-H benchmark ${ }^{\dagger}$. The running example is an ETL process, which extracts data from a source relational database (TPC-H DB) and after processing, loads data to a data warehouse (DW) and can be described by the following query: Load in the DW all the suppliers in Europe together with their information (phones, addresses etc.), sorted on their revenue. The tables that are used from the source database are Supplier, Nation, Region and Lineitem. After Supplier entries have been filtered to keep only suppliers in Europe, the revenue for each supplier is calculated based on the supplied lineitems and subsequently, they are sorted on revenue and loaded to the DW. One simple conceptual model (CM_A) for this process is depicted in Fig. 1, using the Business Process Model and Notation $\left(\mathrm{BPMN}^{\ddagger}\right)$.

An ETL process can be designed in more than one way, each design offering different advantages over the other. In Fig. 2 we can see an alternative conceptual model (CM_B) for our example ETL process with the same functionality as CM_A (Fig. 1), yet including additional tasks. It includes a Web Service (WS) call to complete suppliers' info and improve data completeness; crosschecking with an external data source to correct information and improve data accuracy; replication of the revenue calculation step to improve robustness; and the addition of a recovery point to improve recoverability. In the following sections we will show through our running example how our

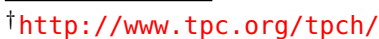

$\ddagger$ http://www. bpmn. org 


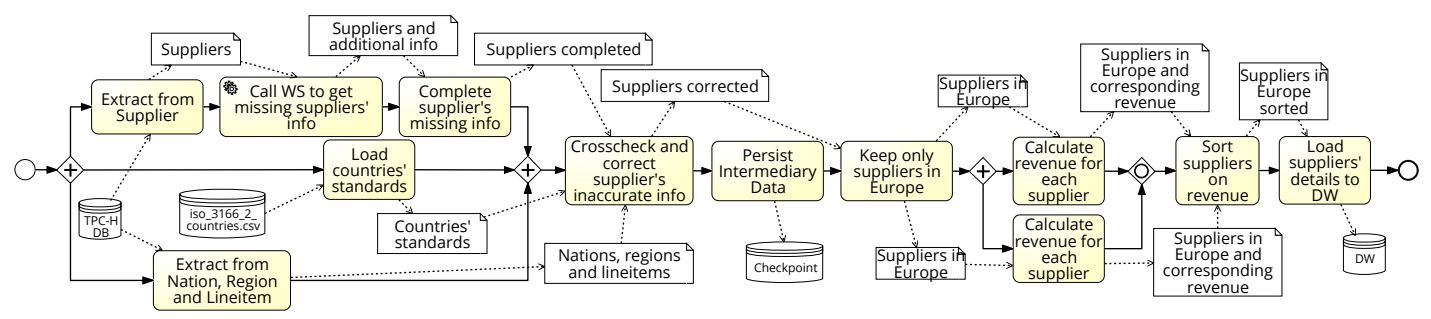

Figure 2. CM_B: Conceptual model of the running example ETL, including additional tasks

proposed models can be used as a basis for i) quality-aware evaluation of ETL processes and ii) ETL process re-design that uses as input only the user-defined importance of different quality characteristics. For the former, we show how the two alternative designs of the same ETL process, despite having the same functionality, differ in quality characteristics in a way that can be quantified, using the measures that we have identified. For the latter, we showcase a user-centered method that starts with the design of CM_A; incrementally generates patterns on the process in order to improve specific quality characteristics; and (semi)-automatically produces the design of CM_B and its corresponding implementation.

The paper is organized as follows. Section 2 presents related work regarding quality characteristics for design evaluation. In Section 3 we present the extraction of our model from related work. The definitions, measures and dependencies among characteristics are presented in Section 4 and Section 5 where we distinguish between characteristics with construct implications and those only for design evaluation, respectively. In Section 6 we present goal modeling frameworks and in Section 7 we validate the usefulness of our models and showcase through examples how they can be used for quality-based ETL process evaluation and redesign. Finally, we provide our conclusions and future work in Section 8.

\section{RELATED WORK}

Quality has always been a central concern for data warehouses, mainly due to the costly resources invested in their development. [10] takes the important step of introducing a business perspective to data warehouse quality, by introducing an extended architecture with different conceptual levels, which enables separation of concerns as well as traceability from client intentions to physical data models. It also captures the notion of goal negotiation for conflicting quality goals.

The significance of quality characteristics for the design and evaluation of ETL processes has also gained attention recently. [6] recognizes the importance of considering not only process functionality but also quality metrics throughout a systematic ETL process design. Thus, it defines a set of quality characteristics specific to ETL processes that it refers to as QoX metrics and provides guidelines for reasoning about the degree of their satisfaction over alternative designs and the tradeoffs among them. A more recent work that has also considered the ideas from [6] is the work from [11]. Based on well-known standards for software quality, the author maps software quality attributes to ETL specific parameters which he calls QoX factors. He defines these factors in ETL context and reasons about the impact that the different ETL subsystems might have on each characteristic.

Focusing on Information Quality, [12] provides a comprehensive list of criteria for the evaluation of Information Systems for data integration. In the same area, [13] identifies most important challenges for Data Integration and highlights quality concerns in distributed, heterogeneous environments. Likewise, [14] identifies the various stakeholders in Data Warehousing activities and the differences in their roles as well as the importance of reasoning among alternative quality concerns and how that affects design choices.

In the last years, there has been an effort in the area of Business Process Management to quantify process quality characteristics and to empirically validate the use of well-defined metrics 
for the evaluation of specific quality characteristics. In this respect, [15] proposes a framework for managing, modeling and evaluating software processes; defines and experimentally validates a set of measures to assess, among others understandability and modifiability of process models. Similar empirical validation is provided by [5], which relates understandability and modifiability to innate characteristics of business process models.

Our approach differs from the above-mentioned ones in that we specifically focus on the process perspective of ETL processes. Instead of providing some characteristics as examples like in [6], we propose a comprehensive list of quality characteristics and we adjust them for our case. In addition, for each of these characteristics we provide quantitative metrics that are backed by literature.

\section{EXTRACTING QUALITY CHARACTERISTICS}

Our model mainly derives from a systematic literature review that we conducted, following the guidelines reported by [16]. The research questions addressed by this study are the following:

RQ1) What ETL process quality characteristics have been addressed?

RQ2) What is the definition for each quality characteristic?

Our search process used an automated keyword search of SpringerLink ${ }^{\S}$, ACM Digital Library ${ }^{\Uparrow}$, ScienceDirect ${ }^{\|}$and IEEE Xplore**. Thus, we searched not one, but multiple electronic resources, following the guidelines from [17]. These electronic resources were chosen because of their popularity within the software engineering community — and the data warehousing research domain in specific - and because we found during our review planning phase that other indexing services (e.g., Citeseer library, Google scholar) only include for our topic of interest, a subset of the material found in our chosen resources. Our goal was to gather all peer-reviewed studies related to our search and therefore we selected a set of resources that is complete with respect to international conference publications, journals and books in the research area of data warehousing.

The search strings were the following:

- (quality attributes OR quality characteristics OR qox) AND ("etl" OR "extraction transformation loading") AND ("information technology" OR "business intelligence")

- (quality attributes OR quality characteristics OR qox) AND ("data integration" OR "information systems integration" OR "data warehouses") AND ("quality aware" OR "quality driven")

The inclusion criterion for the studies was that they should identify a wide range of quality characteristics for data integration processes and thus only studies that mentioned at least 10 different quality characteristics were included. The quality characteristics could refer to any stage of the process as well as to the quality of the target repositories as a result of the process. We should mention at this point that there exists a large number of studies focusing specifically on the quality dimension of Data Quality, but the rationale of our search was to gather all different quality dimensions previously studied, which justifies our inclusion criterion. One exclusion criterion was that studies should be written in English. Whenever multiple studies from same line of work were identified, our approach was to include the most recent or the most extensive version of the study.

The result of our selection process was a final set of 5 studies. Nevertheless, in an attempt to improve the completeness of our sources, we also considered the ETL subsystems as defined in [18] for an industry perspective on the area, as well as standards from the field of software quality. Regarding software quality, our approach was to study the work by [19] and include in our model all the attributes relevant to ETL processes, with the required definition adjustments. This way we reviewed a commonly accepted, generic taxonomy of software quality attributes, while at the same

§http://link.springer.com

I http : //dl . acm . org

\|http://www.sciencedirect.com/

**http://ieeexplore.iee. org 


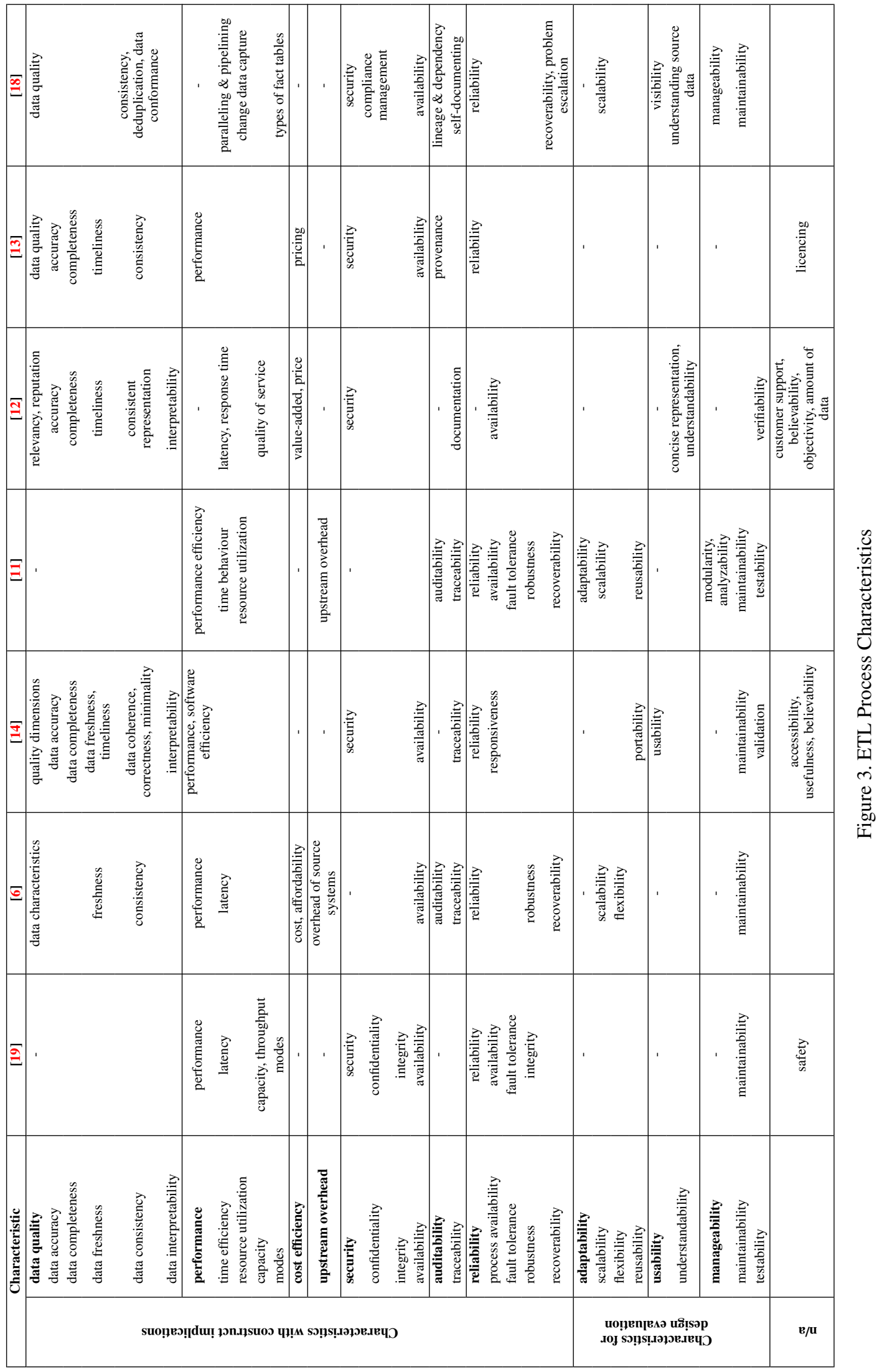


time avoiding the adherence to more recent, strictly defined standards for practical industrial use, which we are nevertheless aware of [20]. The complete list from the resulting 7 sources, covering the most important characteristics from a process perspective can be seen in Fig. 3. In many cases we discovered that the same quality characteristic was referenced using a different term (synonym) and that term is shown at the corresponding table cell under each approach.

Data quality is a prime quality chracteristic of ETL processes. Its significance is recognized by all the approaches presented in our selected sources, except for [11] and [19], since the factors in their analyses derive directly or indirectly from generic software quality attributes. Our model was enriched with a more clear perspective of data quality in Information Systems and a practical view of how quality criteria can lead to design decisions, after reviewing the work by [12]. Although this framework is intended for interoperation of distributed Information Systems in general, many aspects are clearly applicable in the case of ETL processes where data sources can be found in diverse locations and types.

Performance, was given attention by all presented approaches, which was expected since time behavior and resource efficiency are the main aspects that have traditionally been examined as optimization objectives. On the other hand, [11] and [6] were the only approaches to include the important characteristic of upstream overhead. However, [11] does not include security, which is discussed in the rest of the works. The same is true for auditability, which is absent from [19] but found in all other works. Reliability on the other hand, is recognized as a crucial quality factor by all approaches. As expected, the more abstract quality characteristics adaptability and usability are less commonly found in the sources, in contrast with manageability which is found in all approaches except for [13], which does not discuss about intangible characteristics.

Although we include cost efficiency in Fig. 3, in the remainder of this paper this characteristic is not examined as the rest. The reason is that we view our quality-based analysis in a similar perspective as [21], who consider cost as a separate concern to the rest of the attributes, according to which any quality attribute can be improved by spending more resources and it is a matter of weighting the benefits of this improvement to the required cost that can lead to rational decisions. In our case, cost is considered to act as a restriction to the search space of alternative process designs. In addition, we regarded safety as non-relevant for the case of ETL processes, since these processes are computer-executable, non-critical and hence the occurrence of accidents or mishaps is not a concern. Similarly, we considered that the characteristics of accessibility, usefulness, customer support, believability, amount of data and objectivity found in [14] and [12] are not relevant for our case, as they refer to the quality of source or target repositories, yet do not depend on the ETL process. Likewise, licencing [13] refers to concrete tools and platforms while our ETL quality analysis is platform independent.

Thus, we regarded for our models only characteristics that are related to the process perspective of the ETL and other aspects, such as characteristics of the source or target repositories, which are independent of the process, were considered to extend beyond the concern of this study. In this respect, we disregarded ETL quality dimensions specific to the target data warehouse, such as the quality of OLAP modeling (e.g., schemata, hierarchies). Nevertheless, our assumption is that the ETL processes evaluated by our models are functionally correct and produce output corresponding to the target repositories' data modeling. Hence, as we will show in the following sections, our method for process redesign receives as input an ETL process that has been designed respecting the information requirements of the data warehouse.

Through our study we identified that there are two different types of characteristics characteristics that can actively drive the generation of patterns in the ETL process design and characteristics that cannot explicitly indicate the use of specific design patterns, but can still be measured and affect the evaluation of and the selection among alternative designs. In the remainder of this paper we refer to the first category as characteristics with construct implications and to the second as characteristics for design evaluation. 


\section{PROCESS CHARACTERISTICS WITH CONSTRUCT IMPLICATIONS}

In this section, we present our model for characteristics with construct implications. Subsequently, we show the relationships between different characteristics and showcase the use of our model through our running example. The proposed list of characteristics and measures can be extended or narrowed down to match the requirements for specific use cases. Thus, our model is extensible and can constitute a template, generically capturing the quality dimensions of ETL processes and their interrelationships, which can be modified and instantiated per case. The definition of each characteristic can be adjusted; the proposed measures are only mentioned as valid examples that can be extended or replaced by other more appropriate ones; users can decide to use only an excerpt of the model; and the quantitative effect that each characteristic can have to another can differ for different cases. The above points also stand for the model of the following section, about characteristics for design evaluation.

\subsection{Characteristics and Measures}

In this subsection, we provide a definition for each characteristic as well as candidate metrics under each definition, based on existing approaches that we discovered coming from literature and practice in the areas of Data Warehousing and Software Engineering. For each metric there is a definition and a symbol, either (+) or $(-)$ denoting whether the maximization or minimization of the metric is desirable, respectively. Similarly to the quality characteristics, the measures come from the areas of Data Warehousing, ETL, Data Integration and Software Engineering and Business Process Management.

1. data quality $(D Q)$ : the fitness for use of the data produced as the outcome of the ETL process. It includes:

(a) data accuracy: percentage of data without data errors.

M1: \% of correct values [22] (+)

M2: \% of delivered accurate entities [22] (+)

(b) data completeness: degree of absence of missing values and entities.

M1: \% of missing entities from their appropriate storage [23, 22] (-)

M2: \% of non-empty values [22] (+)

(c) data freshness: indicator of how recent data is with respect to time elapsed since last update of the target repository from the data source.

M1: Instant when data are stored in the system - Instant when data are updated in the real world [22] (-)

M2: Request time - Time of last update [22] (-)

M3: 1 / (1 - age * Frequency of updates) [22] (-)

(d) data consistency: degree to which each user sees a consistent view of the data and data integrity is maintained throughout transactions and across data sources.

M1: \% of entities that violate business rules $[23,22](-)$

M2: \% of duplicates [22] (-)

(e) data interpretability: degree to which users can understand data that they get.

M1: \# of entities with interpretable data (documentation for important values) [22] (+)

M2: Score from User Survey (Questionnaire) [22] (+)

2. performance $(P F)$ : the performance of the ETL process as it is implemented on a system, relative to the amount of resources utilized and the timeliness of the service delivered. It includes:

(a) time efficiency: the degree of low response times, low processing times and high throughput rates. 
M1: Process cycle time [24] (-)

M2: Average latency per entity in regular execution [23] (-)

M3: Min/Max/Average number of blocking operations [23] (-)

(b) resource utilization: the amounts and types of resources used by the ETL process.

M1: CPU load, in percentage of utilization [24] (-)

M2: Memory load, in percentage of utilization [24] (-)

(c) capacity: the demand that can be placed on the system while continuing to meet time and throughput requirements.

M1: Throughput of regular workflow execution [23] (+)

(d) modes: the support for different modes of the ETL process based on demand and changing requirements, for example batch processing, real-time event-based processing, etc.

M1: Number of supported modes / Number of all possible modes (+)

3. upstream overhead $(U O)$ : the degree of additional load that the process causes to the data sources on top of their normal operations.

M1: Min/Max/Average timeline of memory consumed by the ETL process at the source system [23] (-)

4. security $(S E)$ : the protection of information during data processes and transactions. It includes:

(a) confidentiality: the degree to which data and processes are protected from unauthorized disclosure.

M1: \% of mobile computers and devices that perform all cryptographic operations using FIPS 140-2 cryptographic modules [25] (+)

M2: \% of systems (workstations, laptops, servers) with latest antispyware signatures [26] (+)

M3: \% of remote access points used to gain unauthorized access [25] (-)

M4: \% of users with access to shared accounts [25] (-)

(b) integrity: the degree to which data and processes are protected from unauthorized modification.

M1: \% of systems (workstations, laptops, servers) with latest antivirus signatures [26] $(+)$

(c) reliability: the degree to which the ETL process can maintain a specified level of performance for a specified period of time. It includes:

i. availability: the degree to which information, communication channels, the system and its security mechanisms are available when needed and functioning correctly.

M1: Mean time between failures (MTBF) [23] (+)

M2: Uptime of ETL process [23] (+)

ii. fault tolerance: the degree to which the process operates as intended despite the presence of faults.

M1: Score representing asynchronous resumption support [23] (+)

iii. robustness: the degree to which the process operates as intended despite unpredictable or malicious input.

M1: \# of replicated processes [23] (+)

iv. recoverability: the degree to which the process can recover the data directly affected in case of interruption or failure.

M1: \# of recovery points used [23] (+)

M2: \% of successfully resumed workflow executions [23] (+)

M3: Mean time to repair (MTTR) [23] (-) 
5. auditability $(A U)$ : the ability of the ETL process to provide data and business rule transparency. It includes:

(a) traceability: the ability to trace the history of the ETL process execution steps and the quality of documented information about runtime.

M1: \% of KPIs that can be followed, discovered or ascertained by end users [27] (+)

\subsection{Characteristics Relationships}

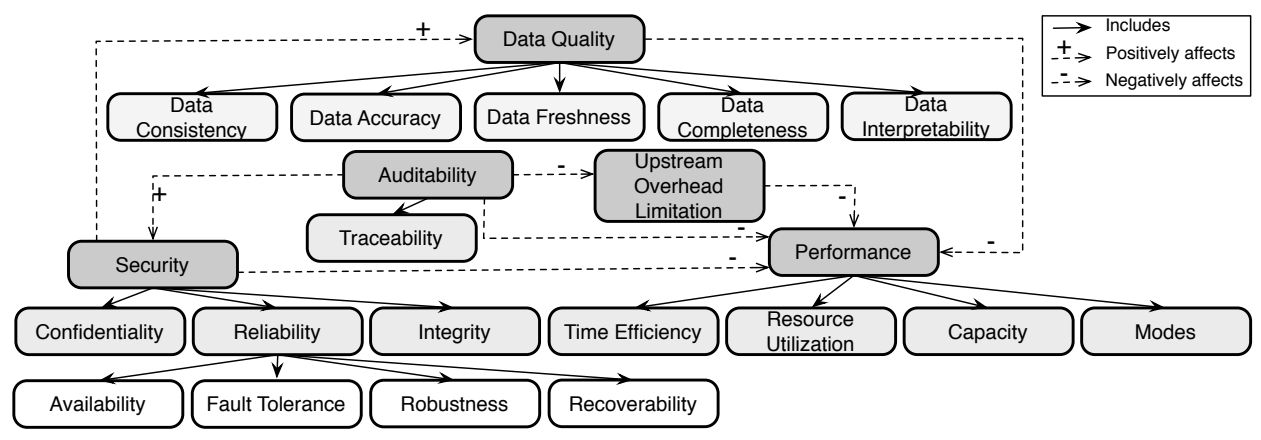

Figure 4. Dependencies among process characteristics with construct implications

In the same direction as [6] and [19] we also recognise that ETL process characteristics are not independent of each other and each time a decision has to be made, the alternative options might affect different characteristics differently, but that this is not realized in completely ad hoc ways. On the contrary, we argue that there is an inherent relationship between characteristics and it can be depicted in a qualitative model that can be instantiated per case for reasoning and automation. Our vision is that this objective model can be generic yet powerful enough in order to aid in a broad range of ETL process design decisions.

Our model for the dependencies among characteristics with construct implications can be seen in Fig. 4. In this model we include all the characteristics with construct implications that we have identified and defined in Sec. 3. It consists of first-level characteristics and in some cases secondor even third-level sub-characteristics and can be read in a cause-and-effect fashion, i.e., improving one characteristic leads to improvement or deterioration of another characteristic. We should notice that although traditionally availability is classified directly under security, for our case availability is in fact a subgoal of reliability. The reason is that we regard the satisfaction of availability as a necessary but not sufficient condition for reliability. Reliability additionally requires maintaining specified SLAs for the ETL process and therefore in our model we place availability under reliability and reliability under security.

Coming back to our running example from Fig. 1 and Fig. 2, it is clear that the second design would require more time and more computational resources than the first one in order to perform the additional tasks. The measures of Process execution time and CPU load measured in percentage of utilization would have higher values indicating worse time efficiency and resource utilization. Thus, improved Data Quality and Reliability would have to be considered at the price of decreased Performance and whether or not the decision to select the second design would be optimal, would depend on the importance of each of these characteristics for the end-user. We will quantitatively show these effects in the following subsection.

As can be seen in Fig. 4 the improvement of any other characteristic negatively affects performance. That is reasonable since such improvements would require the addition of extra complexity to the ETL process, diverging from the optimal simplicity that favours performance. Improving Data Quality would require additional checks, more frequent refreshments, additional data processing and so on, thus utilizing more resources and imposing a heavier load on the system. In the same manner, improving security would require more complex authentication, 
authorization and accounting (AAA) mechanisms, encryption, additional recovery points, etc., similarly having negative impact on performance. Likewise, improving auditability would require additional processes for logging, monitoring as well as more resources to constantly provide realtime access to such information to end-users. In a similar fashion, promoting upstream overhead limitation would demand locks and scheduling to minimize impact of ETL processes on competing resources and therefore time and throughput limitations.

On the other hand, improving security positively affects data quality since data becomes more protected against ignorant users and attackers, making it more difficult for data and system processes to be altered, destroyed or corrupted. Therefore, data integrity becomes easier to maintain. In addition, improved system availability and robustness leads to improved data quality in the sense that processes for data refreshing, data cleaning and so on remain undisrupted.

Regarding the impact that improving auditability has to security, it is obvious that keeping track of system's operation traces and producing real-time monitoring analytics foster faster and easier threat detection and mitigation, thus significantly benefiting security. On the contrary, these operations have a negative impact on upstream overhead limitation, following the principle that one system cannot be measured without at the same time being affected.

\subsection{Calculating the measures}

In this subsection, we go through the measures that we have defined and apply them on our running example. We will do the same for the following section about the second category of measures and for both categories, each measure is represented in the form of:

- $<$ Char_Abbr $>.<$ Subchar_No $>.<\ldots .>$. $<$ Measure_Id $>(<$ Model_Id $>)$

, where Char_Abbr is the characteristic abbreviation (e.g., PF for performance); Subchar_No, Measure_Id etc., are the indexes of the measures' enumerations from the related sections; and $M o d e l \_I d$ can take the values $C M \_A$ or $C M \_B$, for the simple or the more complex ETL flow, or can be missing if we refer to both examples.

Through this example we showcase i) how our proposed measures can be used and ii) how two alternative ETL designs for the same process can differ with regards to different quality dimensions, as is reflected by the corresponding measures, in a way that qualitatively agrees with our proposed model.

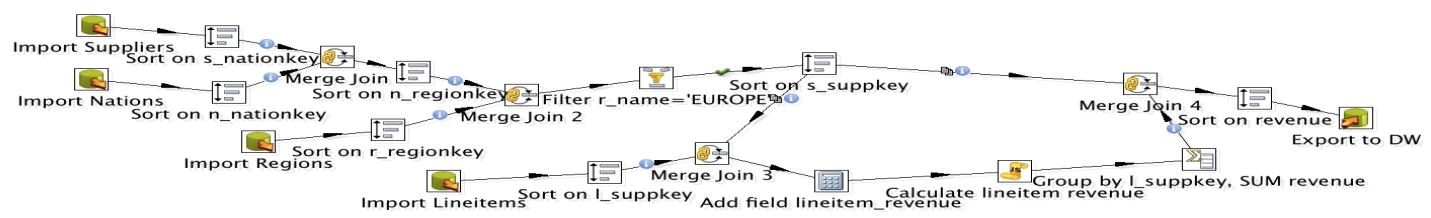

(a) Logical representation of initial ETL process that corresponds to CM_A

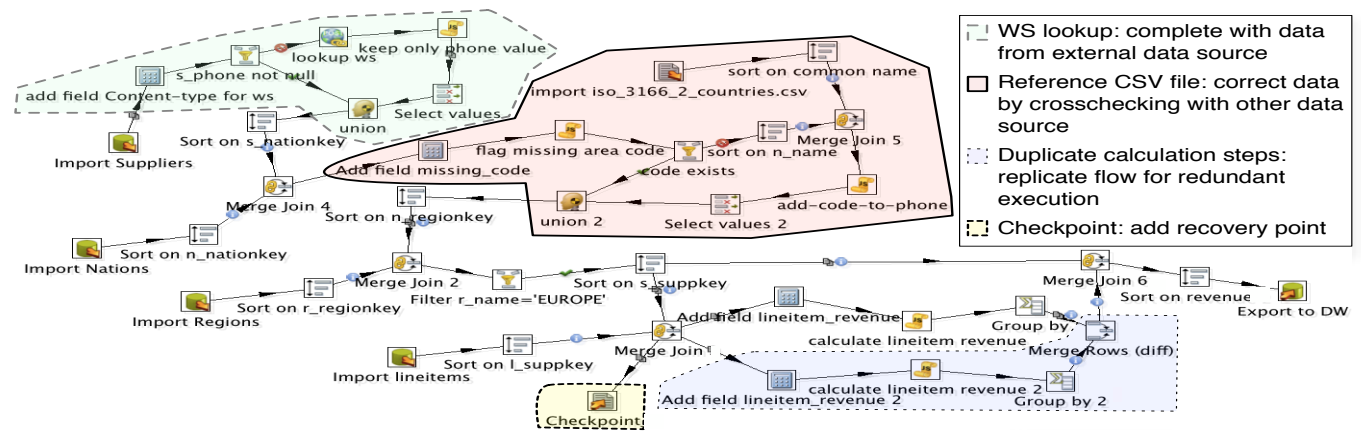

(b) Logical representation of equivalent ETL process with additional steps that corresponds to CM_B

Figure 5. Logical models of alternative ETL processes 
In Fig. 5 we can see the corresponding logical representations of the ETL designs from Fig. 1 and Fig. 2, that were drawn using the Pentaho Data Integration ${ }^{\dagger \dagger}$ Visual Designer. We conducted our experiments using MySQL database that was populated with data generated by the TPC-H data generator. The number of tuples for each table of the database were as follows:

\begin{tabular}{|l|l|l|l|l|}
\hline Table & Supplier & Nation & Region & Lineitem \\
\hline \# of tuples & 10000 & 25 & 5 & 540189 \\
\hline
\end{tabular}

Data quality can be measured for the data produced and exported to the Data Warehouse. Obviously, the quality of these data depends not only on the ETL process, but also on the data quality at the resources that it utilizes, which in our case is the data quality of the TPC-H database, as well as the data quality of the data provided by the WS and the text file, for the second ETL flow. For illustration purposes, we artificially dirtied the automatically generated data on the TCP-H database including null values for the phones of suppliers, as well as phone numbers with wrong formatting, not including country codes. We executed this process randomly, but for a specified percentage of records. We have manipulated the data on the TPC-H database so that they contain null address values for $10 \%$ of all the suppliers, as well as missing area code at the $10 \%$ of non-null phone numbers of the suppliers stored in the database. After executing CM_A or CM_B, the result is the export of 1987 records about suppliers and their revenues to the DW.

To measure data accuracy, we count the number of (non-null) exported records that are incorrect, which for our case translates to missing area codes of phone numbers. Then for each of the two ETL flows we get the values for DQ.a.M1 as shown in Table I.

We notice here that for the second ETL flow, all the incorrect values have been identified and corrected by crosschecking with the text file. That is because we assume that the text file is complete and does not contain any mistakes. This measure could similarly have been used for the incorrect addresses or names if there was a mechanism to identify them or a record could be considered correct only if all the considered attribute values were correct and then the measure could be used in a combined way. The last case would measure the percentage of delivered accurate tuples, as defined in DQ.a.M2 of data accuracy.

For data completeness, we count the number of null values, which for our case can be found in the suppliers' phone number field. Thus, we get the values for DQ.b.M2 as shown in Table I.

Again, we observe that data completeness for the second ETL flow is improved, due to the completion of data using the WS. The reason that completeness for the second ETL flow is not $100 \%$, is because we have created the WS registry so that approximately only half of the suppliers can be found there with their details, to resemble the real case situation where improved completeness could come from a more complete registry, of course at a higher cost. When it comes to measure DQ.b.M1 of data completeness, incompleteness of that kind could occur when incorrect or missing values would result in tuples of suppliers not being present on the final resultset.

Considering data freshness, it would be the same for both ETL flows, unless we assumed that external data sources (WS registry and text file) contain more/less up-to-date information. If we assume for example that the data sources were updated 20 days ago where time units are days, and that they get updated once every month, we can calculate DQ.c.M3 as shown in Table I.

To estimate DQ.c.MI, we would need to know for example when suppliers changed their phone number and when it was updated in the database. For DQ.c.M2 we would need to know how much time passed between the last update of a record and when it was actually first requested.

When it comes to data consistency, DQ.d.M2 could be measured by the percentage of tuples in the resulting dataset that refer to the same supplier, which nevertheless do not exist in our example since every supplier is uniquely included. In addition, DQ.d.M1 could have been affected if there were entries with different names for the same country (e.g., United Kingdom of Great Britain and

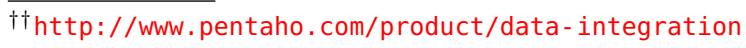


Table I. Data quality measures

\begin{tabular}{|c|c|c|c|}
\hline Measure & DQ.a.M1 & DQ.b.M2 & DQ.c.M3 \\
\hline Formula & $\frac{\text { correct_tupples }}{\text { all_tuples }}$ & $\frac{\text { non_null_tupples }}{\text { all_tuples }}$ & $\frac{1}{1-\text { age*update_frequency }}$ \\
\hline CM_A & $91.5 \%$ & $90.3 \%$ & 3 \\
\hline CM_B & $100 \%$ & $95.2 \%$ & 3 \\
\hline
\end{tabular}

Northern Ireland as opposed to United Kingdom) or values of different format for the same attribute (i.e., phone number as "424-242-424-242" instead of "424242424242").

For data interpretability, to measure DQ.e.M1 we would take under consideration the existence of comment fields for important attributes and their completeness for the resulting data. We notice that there are plenty such comment fields in our database. For DQ.e.M2, we would conduct a survey where actual/potential users of the ETL process would describe how well they understand the meaning and content of each field of the resulting data.

Performance was measured by executing both of the ETL flows on Kettle Engine, running on Mac OS X, 1.7 GHz Intel Core i5, 4GB DDR3 and kept average values from 10 executions.

In Table II we can see the calculation of values for measures PF.a.M1 and PF.a.M3 for time efficiency.

Due to the existence of blocking operators, it is not relevant to calculate measure PF.a.M2 for latency per tuple. As blocking operations, we consider the steps that have to be completed and all of the values that they process have to be calculated before moving to the succeeding operators, which for our case are the aggregation and the sorting steps.

For resource utilization, the calculation of measures PF.b.M1 and PF.b.M2 are shown in Table II and Table III.

For capacity, we consider the size of the input datasets processed by these ETL flows, divided by the time that they need to execute and calculate measures PF.c.M1 in Table III.

We should notice here that the input size for the two processes slightly differs, because for the second process we also have to take under consideration the input data that come from the data cleaning tasks (WS and text file). A possible explanation for the big difference of the throughput values for the two ETL flows can be that the second flow requires the concurrent execution of a number of steps, which decreases the available processing power that accounts for each.

It is clear from all the performance measures shown above, that the design of CM_B is significantly worse that that of CM_A with respect to performance. In other words, the measures agree with our model, according to which the improvement of some quality characteristic(s) (i.e., data quality and security in our example) negatively affects some other(s) (i.e., performance, if we compare CM_B to CM_A).

When it comes to modes, if we consider batch processing and real-time processing as the two possible modes, our ETL flows are only designed to be executed in a batch mode, as calculated in measure PF.d.MI (Table III).

Upstream overhead can be considered as the additional load that comes into play because of the use of additional services, which in our case can refer to the WS. Therefore, there would be upstream overhead only for the second ETL flow and it could be measured as such:

- $U O . M 1\left(C M \_B\right)=A V G \_m e m o r y \_c o n s u m e d \_b y \_W S=426 M B$

This memory consumption lasted for as long as the interaction of the ETL with the WS that was an avarage of $12.9 \mathrm{sec}$, which corresponds to: $\frac{12.9 \mathrm{sec}}{18.9 \mathrm{sec}}=68 \%$ of the process cycle time.

Security did not play an important role for setting up our tests, except from reliability. Thus, for confidentiality, SE.a.M1 would be 0 , since we are not using any cryptographic operations and the web service communication is realized over HTTP. SE.a.M2 would refer to the anti-spyware installed in the operation system of the computer where we run the ETL processes and SE.a.M3 would also be 0 since there was no provisioning for accessing or firing the execution of the ETL processes remotely. SE.a.M4 would be $100 \%$, since all users could have shared access to the 
Table II. Performance measures - Part 1

\begin{tabular}{|c|c|c|c|}
\hline Measure & PF.a.M1 & PF.a.M3 & PF.b.M1 \\
\hline Formula & Process_cycle_time & No_of_aggr + No_of_sort & $\frac{\text { No_of_logical_processors }}{49.25}$ \\
\hline CM_A & $10.4 \mathrm{sec}$ & 8 & 55.75 \\
\hline CM_B & $18.9 \mathrm{sec}$ & 11 & \\
\hline
\end{tabular}

Table III. Performance measures - Part 2

\begin{tabular}{|c|c|c|c|}
\hline Measure & PF.b.M2 & PF.c.M1 & PF.d.M1 \\
\hline Formula & $\frac{\text { used_memory }}{\text { total_memory }}$ & $\frac{\text { Input_data_size }}{\text { Process_cycletime }}$ & $\frac{\text { No_supported_modes }}{\text { all_possible_modes }}$ \\
\hline CM_A & 0.166 & $52906 \frac{\text { tuples }}{\text { sec }}$ & $50 \%$ \\
\hline CM_B & 0.181 & $29179 \frac{\text { tuples }}{\text { sec }}$ & $50 \%$ \\
\hline
\end{tabular}

terminal of the process execution. Similarly for integrity, SE.b.M1 would refer to antivirus installed in the operation system of the terminal where we run the ETL processes.

When it comes to reliability, the measures for availability and fault tolerance can easily be extracted from historic traces of the ETL process execution, monitoring the time of failures and the periods during which the ETL process was available and the periods during which it was not responsive, for example due to memory limitations in high demand. Of course, such analysis presumes that the ETL process has a continuous lifecycle, corresponding to changing data in the data sources. Regarding robustness, in the second ETL flow there is a part that duplicates the calculation steps to guarantee that even in the presence of unpredictable or malicious input that could cause the failure of this part of the ETL flow, there will be a duplicate of this part for the resumption of the execution. Thus, the measure for the second ETL flow is:

- SE.c.iii.M1 $\left(C M \_B\right)=$ No_of_replicated_processes $=1$

In a similar manner, recoverability is improved in the second ETL flow, by the addition of a recovery point. Thus, if for any reason the execution of the ETL flow is interrupted or terminated, already processed data is not lost and execution can continue, starting from the latest checkpoint.

- SE.c.iv.M1(CM_B $)=$ No_of_recovery_points $=1$

The next two measures SE.c.iv.M2 and SE.c.iv.M3 for recoverability, can again be extracted from historic traces of the ETL process execution.

Auditability and traceability can be measured, arguing that all of the KPIs or measures that have been considered so far can be monitored and followed for both ETL flows. Therefore, assuming that these are all the KPIs that we are interested in, the corresponding measure would be:

- AU.a.M1 = \%_of_observable_KPIs $=\frac{\text { No_of_observable_KPIs }}{\text { No_of_important_KPIs }}=\frac{31}{31}=100 \%$

\section{PROCESS CHARACTERISTICS FOR DESIGN EVALUATION}

In this section we show our model about characteristics for design evaluation. These characteristics are the most difficult ones to analyze as they are more abstract and intangible. As mentioned above, the intention of this model is to be used as an extensible, modifiable template that can be adjusted per case. Similarly to Sec. 4 we first define the characteristics and then show the relationships among them.

\subsection{Characteristics and Measures}

In this subsection we provide a definition for each characteristic for design evaluation, as well as proposed metrics deriving from literature. 
1. adaptability $(A D)$ : the degree to which ETL process can effectively and efficiently be adapted for different operational or usage environments. It includes:

(a) scalability: the ability of the ETL process to handle a growing demand, regarding both the size and complexity of input data and the number of concurrent process users.

M1: Ratio of system's productivity figures at two different scale factors, where productivity figure $=$ throughput $*$ QoS/ cost $[28](+)$

M2: \# of Work Products of the process model, i.e., documents and models produced during process execution [15] (-)

(b) flexibility: the ability of the ETL flow to provide alternative options and dynamically adjust to environmental changes (e.g., by automatically switching endpoints).

M1: \# of precedence dependences between activities [15] (-)

(c) reusability: the degree to which components of the ETL process can be used for operations of other processes.

M1: \% of reused low level operations in the ETL process [29] (+)

The following measure is valid in the case where there are statistical data about the use of various modules (e.g., transformation or mapping operations) of the ETL process:

M2: Average of how many times low level operations in the ETL process have been reused per specified time frame [29] (+)

2. usability (US): the ease of use and configuration of the implemented ETL process on the system. It includes:

(a) understandability: the clearness and self-descriptiveness of the ETL process model for (non-technical) end users.

M1: \# of activities of the software process model [15] (-)

M2: \# of precedence dependences between activities [15] (-)

3. manageability $(M N)$ : the easiness of monitoring, analyzing, testing and tuning the implemented ETL process.

(a) maintainability: the degree of effectiveness and efficiency with which the ETL process can be modified to implement any future changes.

M1: Length of process workflow's longest path [23] (-)

M2: \# of relationships among workflow's components [23] (-)

M3: \# of input and output flows in the process model [30] (-)

M4: \# of output elements in the process model [30] (-)

M5: \# of merge elements in the process model [30] (-)

M6: \# of input and output elements in the process model [30] (-)

(b) testability: the degree to which the process can be tested for feasibility, functional correctness and performance prediction.

M1: Cyclomatic Complexity of the ETL process workflow [31] (-)

\subsection{Characteristics Relationships}

In Fig. 6 we show the dependencies among characteristics for design evaluation. Increased usability favors manageability because a more concise, self-descriptive system is easier to operate and maintain. Similarly, adaptability positively affects usability, since an easily configured system is easier to use and does not require specialized skill-set from the end user. On the other hand, adaptability can be achieved with more complex systems and therefore it negatively affects manageability. This negative relationship might appear counter-intuitive, but it should be noted that our view of adaptability does not refer to autonomic behavior, which would possibly provide self-management capabilities. Instead, we regard manageability from an operator's perspective where control is desirable and the addition of unpredictable, "hidden" mechanisms would make 


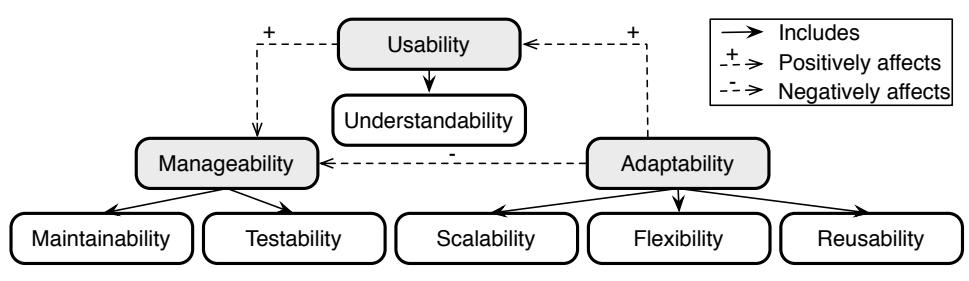

Figure 6. Dependencies among characteristics for design evaluation

the process more difficult to test and maintain. Regarding the apparent conflict between the negative direct relationship among Adaptability and Manageability and the transitive positive relationship between Adaptability, Usability and Manageability, our qualitative model allows for different direct and indirect influences between characteristics. If the model is instantiated and extended with measurable components to quantitatively specify these influences (i.e., weights on the edges of the Digraph), then the compound effect that the improvement of one characteristic has to another will depend on the elicitation techniques used.

\subsection{Calculating the measures}

Similarly to the previous section and using the same notation, we show in this subsection how our measures can be calculated for the characteristics for design evaluation, for the two alternative designs of Fig. 5.

Adaptability was measured as such: for the first measure AD.a.M1 of scalability, we can calculate the throughput, cost and QoS for two different scale factors, for example for different allocation of resources (CPU, memory etc.) to the system for the ETL process execution. As QoS we can consider any of the measures that we have examined so far. The calculation of measure AD.a.M2 is as follows:

- AD.a.M2(CM_A $)=$ No_of_work_products $=1$

- AD.a.M2(CM_B $)=N o \_o f \_w o r k \_p r o d u c t s=2$

The difference is due to the fact that the second ETL flow exports data not only to the DW, but also to the checkpoint and so it has 2 work products.

To measure flexibility, we have to count the precedence dependences between activities, for example for every Join operation, the incoming data streams must be sorted, which dictates precedence dependences between Join and Sort operators. Essentially, the way that both of these ETL flows have been designed, there are precedence dependences between each operation and its predecessor(s), so this measure will equal to the number of edges, if we view the ETL process as a Directed Acyclic Graph (DAG):

- AD.b.M1 $\left(C M \_A\right)=N o \_o f \_p r e c e d e n c e \_d e p e n d e n c i e s=20$

- AD.b.M1 $\left(C M \_B\right)=N o \_o f \_p r e c e d e n c e \_d e p e n d e n c i e s=44$

Regarding reusability, we can calculate measures AD.c.M1 and AD.c.M2 with respect to the ability of operations to be reused in other ETL flows. For example, using the GUI for Pentaho Data Integration (PDI), some operators can simply be copied/pasted to/from the clipboard and function normally as part of different flows. These operators could be considered to have locality among the different flows. This is not true for example for Table input/output operations, since the database connectivity and data source to be used must be explicitly defined for every flow. For measure AD.c.M1, we can compare the two ETL flows and identify the operators that have been reused from the first to the second flow, which for our case are all of the operators from the first flow:

- AD.c.M1 $\left(C M \_B\right)=\%$ \%of_reused_operators $=\frac{\text { No_of_reused_operators }}{\text { No_of_operators }}=\frac{20}{20}=100 \%$

Usability and understandability were evaluated using the following measures: 
Table IV. Maintainability measures - Part 1

\begin{tabular}{|c|c|c|c|}
\hline Measure & $M N . a . M 1$ & MN.a.M2 & MN.a.M3 \\
\hline Formula & Length_of_longest_path & No_of_activity_relationships & No_of_i/o_flows \\
\hline CM_A & 9 & 20 & 5 \\
\hline CM_B & 23 & 44 & 8 \\
\hline
\end{tabular}

Table V. Maintainability measures - Part 2

\begin{tabular}{|c|c|c|c|}
\hline Measure & MN.a.M4 & MN.a.M5 & MN.a.M6 \\
\hline Formula & No_of_outputs & No_of_merge & No_of_i/o_elements \\
\hline CM_A & 1 & 4 & 5 \\
\hline CM_B & 2 & 8 & 8 \\
\hline
\end{tabular}

- US.a.M1 $\left(C M \_A\right)=N o \_o f \_a c t i v i t i e s=20$

- US.a.M2 $\left(C M \_A\right)=N o \_o f \_p r e c e d e n c e \_d e p e n d e n c i e s=20$

- US.a.M1 $\left(C M \_B\right)=N o \_o f \_a c t i v i t i e s=41$

- US.a.M2 $\left(C M \_B\right)=N o \_o f \_p r e c e d e n c e \_d e p e n d e n c i e s=44$

Manageability can be measured, if we consider the ETL flow as a DAG, where each ETL logical operation corresponds to a node and each control flow precedence relationship corresponds to a directed edge. Hence, we have the measures for maintainability as shown in Table IV and Table V.

Regarding testability, we can measure Cyclomatic Complexity (CC) for each flow:

- $M N \cdot b \cdot M 1\left(C M \_A\right)=C C=N o \_o f \_n o d e s-N o \_o f \_e d g e s+N o \_o f \_c y c l e s=$ $20-20+0=0$

- $M N . b . M 1\left(C M \_B\right)=C C=44-41+0=3$

From the above measures, we can clearly see how the design of CM_B is less usable, manageable and adaptable than CM_A. This is not only an intuitive impression from looking at a more complex process model, but can also be quantitatively measured in an automatic and straightforward fashion, thanks to the gathered metrics.

\section{GOAL MODELING FOR ETL DESIGN}

The research area of Requirements Engineering has been active over the past years, offering a plethora of frameworks and methodologies to bridge the gap between stakeholders' (early and late) design goals and specific decisions. The field of Goal-Oriented Requirements Engineering additionally depicts the alignment between requirements and goals and their relationship to strategic decisions.

A goal constitutes an objective of a business, for example Increase revenue, Improve customer satisfaction and so on. A goal can be satisfied or not, depending not only on the success of the corresponding tactical steps undertaken but also on factors external to the goal, such as environmental conditions or the satisfaction or not of other goals that can affect it. Hence, a goal can be decomposed (or refined as found in literature) to other more simple goals - its subgoals in different ways, the most common being AND and OR decomposition. Examples of subgoals for the goal Increase revenue, can be the goals Reduce production costs and Increase sales.

In general, the semantics of an AND-decomposition is that if all of the subgoals of a goal $G$ that participate in the same AND-decomposition are satisfied, then $G$ is satisfied. Regarding ORdecomposition, it introduces the possibility of alternative ways to satisfy one goal. That is, if any of the (groups of AND-)subgoals of a goal $G$ that participate in an OR-decomposition is satisfied, then $G$ is satisfied. Furthermore, the satisfaction of a goal might be influenced by the satisfaction of another goal, either positively or negatively. 
It is natural that goals and their interrelationships are usually depicted diagrammatically using goal-models, with goals being represented as boxes and arrows connecting them to show relationships for goal elicitation. For our case, goals are quality goals about the ETL process (e.g., Improve performance, Improve data quality) and as we have mentioned above, the satisfaction of one such goal might affect negatively or positively the satisfaction of another, which can be shown in the model using arrows of positive or negative influence. In addition, quality goals can be decomposed to subgoals, for example the goal Improve reliability can be satisfied either by satisfying the goal Improve robustness OR the goal Improve recoverability. These refinements can be directly derived by our model of quality characteristics and subcharacteristics, where the improvement of the latter can play the role of subgoals to the goals of improvement of the former. We can see for our use case, goals, subgoals and influences in Fig. 7, but more details about this model will be provided below, where we describe the specific goal modeling paradigm that has been used.

Goal models are used not only to lighten for business users the cognitive load of managing multiple goals but also to aid in their analysis through reasoning. By defining specific rules for satisfaction propagation through different parts of the goal model (e.g., if any of the AND-subgoals is denied $\rightarrow$ the goal is denied), users can assume given values for part of the model and test the feasibility of different what-if scenarios; they can see what implications (if any) are applied to the other parts of the model; in some models they can even make decisions about specific actions to be taken, in a top down approach where they select which goal(s) they would like to satisfy. Different goal modeling paradigms offer different levels of expressiveness.

A thorough review of goal-oriented approaches is provided by [32,33] which present the basic concepts of goal-oriented requirements engineering and show through examples how goals can be refined for the selection among alternative architectural designs. The author illustrates the use of the KAOS framework for modeling, specifying and analyzing requirements in order to decide about system architectures. Additionally to goal decompositions, contribution links and partial negative or positive contribution among goals, the NFR (non-functional requirement) modeling framework [34] also introduces the concept of softgoals: intangible goals without clear-cut criteria. The $\mathrm{i}^{*}$ [35] framework integrates these concepts with resources, and dependencies between actors to facilitate the modeling for identifying stakeholders and for examining the problem domain while exposing early phase system design requirements.

In a more recent work, [36] compares several existing approaches on goal oriented requirements analysis and shows how different modeling of the same goals can lead to different evaluation and decisions.

Goal modeling approaches have also been suggested to aid with decision making for Business Intelligence. An application of the Tropos goal modeling methodology [37] to data warehousing is suggested by [38], which introduces a technique to map requirements with facts, dimensions and measures to relevant data schemata and decisions. This technique can be seen as a first step towards requirements-oriented data warehousing, concentrating on functionality and early phase design. [39] presents a goal modeling framework to support Collaborative BI systems requirements elicitation and the business intelligence model (BIM) [40] is a goal modeling paradigm, specific to BI context that is used to represent, in an object-centered way the interaction among different goals and situations.

\subsection{Applying BIM to ETL processes}

We applied the BIM Model for our running example (Fig. 7), in order to aid in ETL Process design decisions. In the BIM model, a goal is an intentional situation that is desired by the (viewpoint) organization and BIM models the positive or negative relationship among them, their elicitation from other goals (subgoals) and from tasks (or processes as they are referred in newer versions), which are processes or set of actions that are related to some goal and can achieve it, to provide a "how" dimension. The satisfaction of a goal can be inferred from the satisfaction level of other goals. For our case, goals are quality goals for the ETL process (e.g., Improve reliability) and tasks are 
some patterns that can be applied to the ETL process in order to improve some quality dimensions (e.g., Add recovery point to improve recoverability).

The BIM core also includes indicators to evaluate the satisfaction of goals and measure the use of processes. Indicators act as the measurable component to bridge the gap between business objectives and real, actual data that support or decline their satisfaction. For our case, our defined quality measures (the actual metrics) from Section 4 and Section 5, can play the role of indicators (e.g., \% of correct values). It also includes internal and external situations to model state information and at the lowest refinement level. For our case, situations can represent states internal or external to the ETL process, which can be favorable or unfavorable to the goals (e.g., an external situation to the ETL process can be the Quality of $H W / S W$ resources).

To put it all together, the main elements of the goal model can be derived in a very straightforward and intuitive manner from the classification and the models that we have introduced in Section 4 and Section 5.

- The improvement of each quality characteristic constitutes a goal.

- The improvement of characteristic $a$ that is a subcharacteristic of characteristic $b$ constitutes an OR-subgoal of the goal Improve b.

- The positive/negative influences between goals can be directly derived from the positive/negative influences that we have shown in the characteristics relationships (Fig. 4 and Fig. 6).

- The measures (metrics) that we have defined for each quality characteristic can play the role of indicators, evaluating the goals related to the corresponding characteristics. It should be noted here that indicators must be unique for each goal, according to the specification of BIM. Thus, in cases where there is more than one measure that can be used for the evaluation of a goal, they should be aggregated (e.g., using simple additive weighting method) into one compound indicator.

Unlike the above-mentioned elements, the tasks and the situations are not directly derived from our models. Tasks represent an arsenal of possible (reusable) actions that can be taken and their definition as well as their impact on goals require domain knowledge and evidence to be supported. For our case, the tasks depicted in our model have been collected from literature and validated by experiments that we have conducted with multiple ETL processes. They have also been translated to patterns in order to be automatically integrable to any ETL flow using our tool implementation, about which we discuss in the following section. Modeling of situations also requires domain knowledge, as well as contextualization to match the state information of specific use cases. This stands not only for tasks and situations, since for different use cases and contexts, different quality characteristics and metrics can be used, which can be different subsets of the elements of our models, and/or different, new ones defined in a similar fashion, thus resulting in different goal models.

As mentioned above, apart from concise visual representation, goal models are used for what-if analysis and reasoning, which for the case of the BIM model is straightforward, since it can be directly translated to the OWL 2 DL [41]. As example of translation of (Fig. 7) to OWL we show the following facts:

Class: ImproveDataQuality SubClass0f: Goal and OR Thing

Class: ImproveDataCompleteness SubClass0f: (refines some ImproveDataQuality)

Class: ImproveDataCompleteness SubClass0f: Goal and AND_Thing

Class: ReduceTuplesWithNullvalues SubClass0f: (refines some ImproveDataCompleteness)

Class: ImproveDataQuality SubClass0f: (-_influences some ImprovePerformance)

Class: ImprovePerformance SubClass0f: (-_infBy some ImproveDataQuality)

Class: ImproveReliability SubClass0f: (+_influences some ImproveDataQuality) 
Class: ImproveDataQuality SubClassof: (+_infBy some ImproveReliability)

Class: ImproveDataQuality SubClass0f: (refinedBy exactly 2) and (refines exactly $\odot$ ) and (influences exactly 1) and (infBy exactly 2)

Listing 1: OWL facts for running example

For more information about the BIM and its translation to OWL, interested readers are referred to [42].

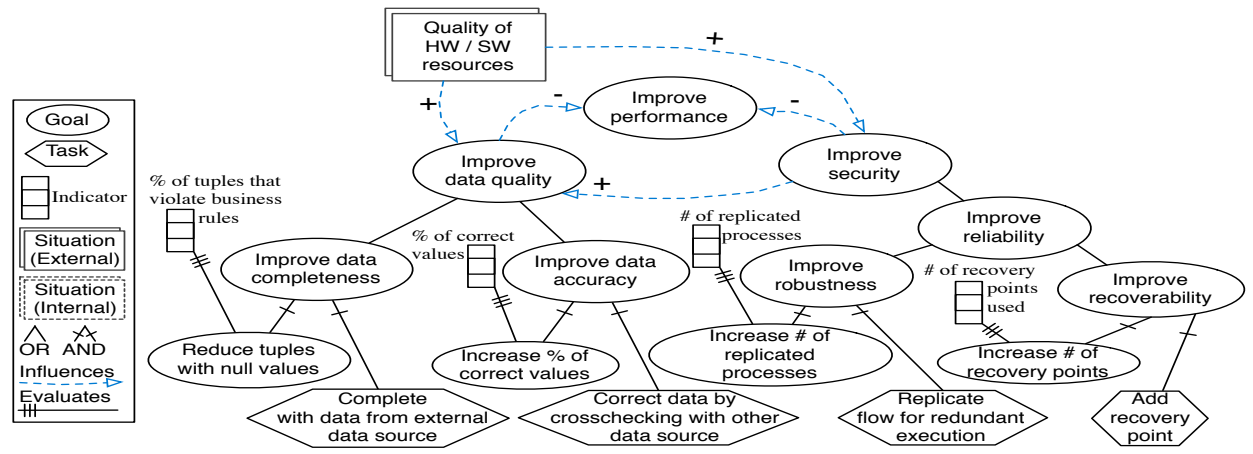

Figure 7. Goal modeling for running example

\section{USER-CENTERED ETL OPTIMIZATION}

In this section, we showcase how our model can provide the conceptual and practical base, upon which a user-centered method can be applied to translate user goals about ETL process quality to specific implementation steps. Thus, we introduce our proposed architecture of this method together with explanation of how it is applied to our running example.

In Fig. 8 we can see the architecture of a novel methodology, which we have presented in [43], for the end-to-end design of ETL processes that takes under consideration both functional and nonfunctional requirements. As can be seen from Fig. 8, which is labeled with a number for each step, our methodology consists of three phases: design of an ETL process based on functional requirements (steps 1-3); improvement by instillation of user-defined quality characteristics to the process (steps 4-13); and finally deployment and execution (steps 15-17). In order to promote automation and user-centricity, our modular architecture employs reuse of components and patterns to streamline the design. Furthermore, for the improvement phase we apply an iterative model where users are the key participants through well-defined collaborative interfaces. In the following we describe each component in more detail, as well as the application to our running example.

\subsection{Functionality-Based Design}

The ETL Process Designer component is responsible for the design of an ETL process model that implements the basic ETL functionality: extraction of data from the original data sources, transformation of data to comply with functional requirements and finally loading into target repositories. This part of the method (steps 1-3 in Fig. 8) only concerns the functional requirements for the ETL process, producing an ETL model that complies with the information requirements and the characteristics of the target repositories (e.g., star schema with specific facts and dimensions). Thus, it is not connected to our models about quality (or non-functional) requirements. However, it is presented here to showcase how it guarantees the generation of a functionally correct ETL process, which can subsequently be provided as input to and be improved by a quality-enhancement phase. 


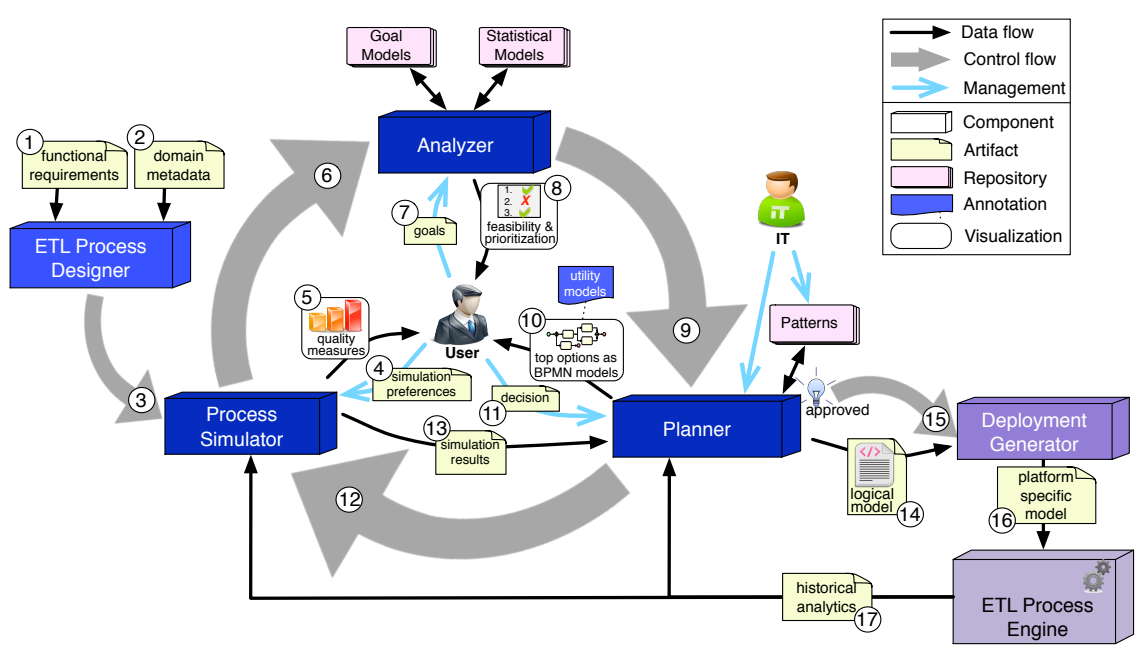

Figure 8. Functional architecture

Recently, several approaches have been proposed for the automation of this phase. For example, [44] use an ontology of the data sources and their functional dependencies, together with business queries, to semi-automatically generate the ETL steps and the data warehouse multi-dimensional model at a conceptual level. Similarly, [45] propose an approach where the domain model along with user-requirements are modeled on the ontological level and subsequently, an ETL process is produced, also modeled as an implementation-independent ontology.

Apparently, the required input at this step is an accurate representation of the domain, covering information not only about available data sources, data schemata, entities and interrelations among them (step 2) but also about business requirements (step 1). Concerning our running example, the input would be a description of the TPC-H relational source tables - schemata and constraints - as well as the appropriate modeling of the query: Load in the DW all the suppliers in Europe together with their information (phones, addresses etc.), sorted on their revenue, for example using description logics or a structured description (XML, JSON etc.).

The output of this step is a conceptual ETL process model, which is described in a high-level representation. This model must be abstract enough to allow for the incorporation of patterns reflecting user requirements, but at the same time it can be seamlessly translated to a logical, implementation independent model. In addition, this model must be directly translatable to an intuitive visualization for the system user, using for example BPMN. Thus, we suggest that the model at this step is an ETL-specific extension of the Directed Acyclic Graph, where each node is one high-level ETL operator. [46] provide such a set of high-level ETL operators as part of their proposed BPMN meta-model. The high level representation output of our running example at this point, is the BPMN process in Fig. 1 and the translation to a logical model is the process in Fig. 5a.

Apart from the process model, domain information about available data sources, entities and their characteristics as well as resource constrains is also passed on to the next phase (step 3) to allow for design alterations, where needed.

\subsection{Quality Enhancement}

The second phase (steps 4-13 in Fig. 8) regards the infusion of quality parameters to the ETL process. Our architectural design at this stage is influenced by two paradigms from the areas of Software Development and Business Intelligence: agile methods and self-service BI [8], respectively. The benefits of using agile methods as opposed to the traditional waterfall approach in Data Warehousing activities have recently been recognized [7]. We identify this stage of the ETL process design as a perfect candidate for the application of agile practices because of the complexity and uncertainty of translating quality requirements to design choices. Thus, we adopt the idea of 
incremental and iterative design with users in the center of the process. Likewise, we adopt the concept of strategy-driven business process analysis from the area of self-service BI, where users make decisions in a declarative fashion based on strategies, goals and measures.

Integrating these ideas, we suggest that users make decisions in stepwise iterations (sprints) that incrementally improve the quality of the ETL process, until they consider it crosses an acceptable quality threshold. Following is a description of each component that shows the means to facilitate this interaction.

The Process Simulator component is assigned with the task of simulating the ETL process and producing primal and complex analytics about both its static structure and its predicted execution behaviour. At this point the users provide their simulation preferences (step 4) that reflect quality characteristics of interest and receive as feedback a user-friendly representation of quality measures (step 5). To this end, it is important to use representative, realistic input data to test the process, which can be achieved either by "feeding" the simulator with a sample of real input data or by providing an effective synthetic test data generation mechanism [47].

Coming back to our running example, the users decide which of the quality characteristics that we have defined in our models they would be interested in, e.g., the quality dimensions of performance, data quality and security. They are also able to browse through and select among predefined metrics that are related to those dimensions and are of interest for their analysis. Such examples are the indicators of the goal model in Fig. 7 and as explained above, ad-hoc, compound, aggregated measures for specific business needs are also used, with the capability of being broken down to more simple metrics, where visualization plays a key role for their presentation to the user. Calculating the measures as has been showcased in previous sections, happens in a completely automated way, thanks to the machine-readable modeling of the ETL process.

One decision that can be made at this point is the level of detail of the simulation. For example, the analysis can take place on a process level or on a task level, based on a single process run or on aggregated results of multiple process replications. Additionally, the simulation methodology can also be decided, especially for the case of loops, conditions and events (e.g., probabilistic or deterministic). The metrics as well as the user input should be applicable for different parts and levels of detail of the process.

Once the measures calculations about the current version of the process have been presented to the users, they can evaluate how well they align with the strategic goals that have been set and decide which of the quality dimensions should be improved, that can be directly translated to goals in the goal modeling of the following step.

Subsequently (step 6), the Analyzer takes as input user goals (step 7) and it is responsible for reasoning about which goals and solution directions are feasible as well as which ones are most fit for use in the specified context. For the first part, it employs goal modeling techniques. As mentioned above, apart from concise visual representation, goal models are used for "what-if" analysis and reasoning. Selecting which goals are pursued every time, goal models can allow to answer feasibility questions about the set of tasks that can be performed, forming the palette of quality patterns that will be used for the optimization problem (step 8).

Considering our running example, the goal model of Fig. 7 can be used for such analysis. For example, the BIM tool ${ }^{\ddagger \ddagger}$ can provide "what-if" analysis regarding how given input information about goals, processes, situations, indicators, and domain assumptions propagates to other elements in the model. For example, it can answer questions such as: what happens (in other words, what are the possible satisfaction values) with the goal "Improve Performance", if we assume that the goal "Increase \# of replicated processes" is satisfied? For the model of Fig. 7 the answer to such questions might appear rather obvious, but this is not the case with real use cases that can produce a very complex business model with tens of different quality characteristics. The situation can become even more complex if we assume some bigger granularity i) in the influences between goals (e.g., using additionally ++ and -- to denote greater positive and negative influence, respectively) and

$\overline{\ddagger \ddagger h t t p: / / W W W . c s}$. utoronto.ca/ jm/bim/ 
ii) in the possible satisfiability values (e.g., using additionally partial satisfaction and partial denial of goals).

A top-down reasoning is also possible, where the user can select which goals need to be satisfied/denied and the tool can provide possible tasks that can be implemented. For example, selecting the satisfaction of the goals Improve security and Improve performance (at the same iteration) would return no possible tasks, but selecting the satisfaction of the goal Improve data quality would return the possible tasks of Complete with data from external data source and Correct data by crosschecking with other data source.

The second process that can be conducted by the Analyzer is the qualitative evaluation of alternative design patterns application. For this purpose, statistical models can be used that will take as input user goals and quality measures from the simulation of alternative ETL process models and will produce as output (step 8) the (quantitative) relationships between goals and quality patterns, and thus the prioritization of the patterns that should be used, based on user's goals.

The Planner is a core component of the quality enhancement phase, responsible for applying patterns on the ETL process that improve its quality, using as input information about the process structure, current estimated metrics and goals and available patterns prioritization. The available pattern toolset can be predefined and extended on a per case basis and the resulting model after the integration of patterns is a logical model. This model includes a set of configuration and management operations that are not directly related to the functionality of specific flow components, but are rather external to the process (e.g., security configurations). These operations are necessary to complete the palette of available improvement steps for the satisfaction of quality goals. For our running example, such patterns are the tasks of the goal model (Fig. 7).

Even though the problem space is restricted by estimated (monetary) cost, the optimization problem of selecting an optimal combination of patterns to be applied to the process can be formulated as a multi-objective knapsack problem [48]. In order to tackle complexity, we propose the use of goal models and statistical models on the previous step on one hand; and the application of only one pattern during each iteration, on the other. In this direction, after reasoning, the Planner recommends to the user a list of the highest ranked potential patterns (step 10) in a graph-like visualization, together with utility models, which are annotations denoting the estimated affect of each pattern to the quality goals. Judging solely from the BPMN models and the utility models, users make a selection decision (step 11) and the Planner implements this decision by integrating a pattern to the existing process flow. These patterns are in the form of process components and the Planner carefully merges them to the existing process [49]. Subsequently, new iteration cycles commence (step 12), until the users consider that the model adequately satisfies quality goals. The Planner receives feedback from the actual runtime of the executed process as well as from their simulation (step 13) in order to adjust its heuristics and increase accuracy when selecting top options.

Assessing the feasibility of our approach, we have implemented and presented POIESIS [50] as a prototype of the Planner component. Our tool offers a predefined set of patterns that can be generated and applied to any ETL process on a logical level. It also offers to users the option of defining their own patterns, reusing existing structures and patterns as templates, as well as their own (compound) measures. Hence, POIESIS takes as input the logical representation of an ETL process, as well as the selected patterns to be used and produces alternative ETL flows with different positioning and combinations of the patterns on each flow. The measures for the quality attributes of interest are presented to the user for the top performing flows (using simulation results) and the user selects which of the alternative flows to be used for implementation or further analysis.

Regarding our running example, after four iterations, where the user would select the goals to Improve data completeness, Improve data accuracy, Improve robustness and Improve reliability, the resulting ETL logical model would be the one of Fig. 5b, which corresponds to the initial ETL model with added and integrated patterns.

For data completeness, a pattern has been added to complete missing rows and null values from external data sources. We implemented a simple web application that receives a (HTTP) request containing the suppliers' names for suppliers with empty (null) values for their phones. After matching those names to existing records in its registry, if found the application replies with 
information about the suppliers, which contains their phones. The corresponding logical steps of the ETL process using this service as a client, can be seen in the WS lookup part of the ETL flow in Fig. 5b. Likewise, for data accuracy, a pattern has been applied to correct data, according to crosschecks with other data sources. We realized this pattern by using a local text file (CSV) that contained the ISO 3166-2 standard information for all countries. This file was crosschecked with the suppliers' records that have missing phone country codes and the corresponding codes for the suppliers' countries were filled in to the telephone numbers. The related logical steps of the ETL process can be seen in the Reference CSV file part of the ETL flow in Fig. 5b. Similarly, to improve reliability and robustness, corresponding patterns of logical steps of the ETL flow, can be seen in the Duplicate calculation steps and the Checkpoint parts of the ETL flow in Fig. 5b, respectively. It should be noticed that the logical steps of the patterns have been generically defined within our tool, resulting in highly configurable patterns that can be integrated to any ETL process with the appropriate configurations (e.g., URLs of available services, attributes to be joined).

\subsection{Deployment and Execution}

Once users observe satisfactory estimations for their measures of interest, they will decide that the quality of the process is acceptable and thus it is ready for deployment and execution (steps 14-17 in Fig. 8). The Deployment Generator component processes the logical model and translates it to a platform-specific model (step 16). This step can be realized using existing approaches for (semi)automated transition among different abstraction levels, focusing on cost and performance [51,3]. The ETL Process Engine executes the ETL process and as mentioned above, keeps traces to provide related historical analytics to the Planner and the Process Simulator (step 17).

\section{SUMMARY AND OUTLOOK}

The automation of ETL processes is a promising direction in order to effectively face emerging challenges in Business Intelligence mainly caused by data volume, velocity and variety. Although information systems are developed by professionals with technical expertise, it is important to align the design of underlying processes with an end-user perspective that reflects business requirements. In this paper, we have proposed a model for ETL process quality characteristics that constructively uses concepts from the fields of Data Warehousing, ETL, Data Integration, Software Engineering and Goal Modeling. One important aspect about our model is that for each and every characteristic, there has been suggested measurable indicators that derive solely from existing literature. Our model includes the relationships between different characteristics and can indicate how the improvement of one characteristic by the application of design modifications can affect others. We have shown how our defined models can be used to automate the task of selecting among alternative designs and improving ETL processes according to defined user goals. Future work will include the definition of methodologies that use our models as staring points to generate various goal models from different frameworks, for which refinements, satisfiability propagation and elicitation of goals will be straightforward.

\section{ACKNOWLEDGEMENT}

This research has been funded by the European Commission through the Erasmus Mundus Joint Doctorate "Information Technologies for Business Intelligence - Doctoral College" (IT4BI-DC).

\section{REFERENCES}

1. Simitsis, A., Vassiliadis, P., Sellis, T. Optimizing ETL processes in data warehouses. In: ICDE. pp. 564-575 (2005)

2. Böhm, M., Wloka, U., Habich, D., Lehner, W. GCIP: Exploiting the generation and optimization of integration processes. pp. 1128-1131. EDBT, ACM (2009)

3. Wilkinson, K., Simitsis, A., Castellanos, M., Dayal, U. Leveraging business process models for ETL design. pp. 15-30. ER, Springer-Verlag (2010) 
4. Akkaoui, Z., Mazón, J.N., Vaisman, A., Zimányi, E. BPMN-based conceptual modeling of ETL processes. In: DaWaK. pp. 1-14. Springer (2012)

5. Sánchez-González, L., García, F., Ruiz, F., Mendling, J. Quality indicators for business process models from a gateway complexity perspective. Inf. Softw. Technol. 54(11), 1159-1174 (2012)

6. Simitsis, A., Wilkinson, K., Castellanos, M., Dayal, U. QoX-driven ETL design: Reducing the cost of ETL consulting engagements. pp. 953-960. SIGMOD, ACM, New York, NY, USA (2009)

7. Golfarelli, Matteo, Rizzi, Stefano, Turricchia, Elisa. Sprint Planning Optimization in Agile Data Warehouse Design. In: DaWaK, pp. 30-41, 2012.

8. Berthold, Henrike, Rösch, Philipp, Zöller, Stefan, Wortmann, Felix, Carenini, Alessio, Campbell, Stuart, Bisson, Pascal, Strohmaier, Frank. An Architecture for Ad-hoc and Collaborative Business Intelligence. In: EDBT, pp. 1-6, 2010.

9. van Lamsweerde, A. Goal-oriented requirements engineering: a guided tour. In: Requirements Engineering. pp. 249-262 (2001)

10. Jarke, Matthias, Jeusfeld, Manfred A., Quix, Christoph, Vassiliadis, Panos. Architecture and quality in data warehouses: An extended repository approach. In: Information Systems 24(3), pp. 229-253 (1999)

11. Pavlov, I. A QoX model for ETL subsystems: Theoretical and industry perspectives. pp. 15-21. CompSysTech, ACM (2013)

12. Naumann, F. Quality-driven Query Answering for Integrated Information Systems. Springer-Verlag (2002)

13. Dustdar, S., Pichler, R., Savenkov, V., Truong, H.L. Quality-aware service-oriented data integration: Requirements, state of the art and open challenges. SIGMOD 41(1), 11-19 (2012)

14. Jarke, M., Lenzerini, M., Vassiliou, Y., Vassiliadis, P. Fundamentals of Data Warehouses. Springer (2003)

15. García, F., Piattini, M., Ruiz, F., Canfora, G., Visaggio, C.A. FMESP: Framework for the modeling and evaluation of software processes. pp. 5-13. QUTE-SWAP, ACM (2004)

16. Kitchenham, B., Pearl Brereton, O., Budgen, D., Turner, M., Bailey, J., Linkman, S. Systematic literature reviews in software engineering - a systematic literature review. Inf. Softw. Technol. 51(1), 7-15 (2009)

17. Pearl Brereton, Barbara A. Kitchenham, David Budgen, Mark Turner, Mohamed Khalil. Lessons from applying the systematic literature review process within the software engineering domain. Journal of Systems and Software $80(4), 571-583$ (2007)

18. The subsystems of ETL revisited. http://Www. informationweek.com/software/information-management/ kimball - university - the - subsystems - of - etl - revisited/d/d - id/1060550 (cited January 2014)

19. Barbacci, M., Klein, M., Longstaff, T., Weinstock, C. Quality Attributes. Tech. rep., Carnegie Mellon University, Pittsburgh, Pennsylvania (1995)

20. Al-Qutaish, R. An investigation of the weaknesses of the ISO 9126 Intl. Standard. In: ICCEE. pp. 275-279 (2009)

21. Kazman, R., Asundi, J., Klein, M. Quantifying the costs and benefits of architectural decisions. In: ICSE. pp. 297-306 (2001)

22. Batini, C., Cappiello, C., Francalanci, C., Maurino, A. Methodologies for data quality assessment and improvement. ACM Comput. Surv. 41(3), 1-52 (2009)

23. Simitsis, A., Vassiliadis, P., Dayal, U., Karagiannis, A., Tziovara, V. Performance evaluation and benchmarking. Lecture Notes in Computer Science vol. 5895, chap. Benchmarking ETL Workflows, pp. 199-220. Springer-Verlag (2009)

24. Majchrzak, T.A., Jansen, T., Kuchen, H. Efficiency evaluation of open source ETL tools. pp. 287-294. SAC, ACM, New York, NY, USA (2011)

25. Chew, E., Swanson, M., Stine, K.M., Bartol, N., Brown, A., Robinson, W. Performance Measurement Guide for Information Security. Tech. rep. (2008)

26. KPILibrary. http://kpilibrary.com (cited January 2014)

27. Leite, J., Cappelli, C. Software transparency. Business and Information Systems Engineering 2(3), 127-139 (2010)

28. Jogalekar, P., Woodside, M. Evaluating the scalability of distributed systems. Parallel and Distributed Systems, IEEE Trans. on 11(6), 589-603 (2000)

29. Frakes, W., Terry, C. Software reuse: Metrics and models. ACM Comput. Surv. 28(2), 415-435 (1996)

30. Muñoz, L., Mazón, J.N., Trujillo, J. Measures for ETL processes models in data warehouses. pp. 33-36. MoSE+DQS, ACM (2009)

31. Gill, G., Kemerer, C. Cyclomatic complexity density and software maintenance productivity. Soft. Eng., IEEE Trans. on 17(12), 1284-1288 (1991)

32. A. van Lamsweerde. Goal-oriented requirements engineering: a guided tour. In: Requirements Engineering, 2001. Proceedings. Fifth IEEE International Symposium on, pages 249-262, 2001.

33. A. van Lamsweerde. From system goals to software architecture. In: Formal Methods for Software Architectures. Volume 2804 of Lecture Notes in Computer Science, pages 25-43. Springer-Verlag, 2003.

34. Chung, Lawrence, Nixon, Brian, Yu, Eric, Mylopoulos, John. Non-Functional Requirements in Software Engineering. In: International Series in Software Engineering, Volume 52000.

35. Eric S. K. Yu. Modelling Strategic Relationships for Process Reengineering. Ph.D. Dissertation, University of Toronto, Toronto, Ont., Canada, 1996.

36. Jennifer Horkoff and Eric S. K. Yu. Comparison and evaluation of goal-oriented satisfaction analysis techniques. In: Requir. Eng., 18 (3): 199-222, 2013.

37. Bresciani, P., Perini, A., Giorgini, P., Giunchiglia, F., Mylopoulos, J. Tropos: An agent-oriented software development methodology. Autonomous Agents and Multi-Agent Systems 8(3), 203-236 (2004)

38. Giorgini, P., Rizzi, S., Garzetti, M. GRAnD: A goal-oriented approach to requirement analysis in data warehouses. Decision Support Systems 45(1), 4 - 21 (2008)

39. Yu, Eric, Dobbie, Gillian, Jarke, Matthias, Purao, Sandeep CSRML4BI: A Goal-Oriented Requirements Approach for Collaborative Business Intelligence. In: Conceptual Modeling, Lecture Notes in Computer Science 8824: 423-430, 2014. 
40. Horkoff, Jennifer, Barone, Daniele, Jiang, Lei et al. Strategic business modeling: representation and reasoning. In: Software \& Systems Modeling 13(3), 1015-1041 (2012)

41. OWL 2 Web Ontology Language Manchester Syntax. http://WWw. W3 . org/TR/owl2-manchester-syntax/ (cited August 2015)

42. Horkoff, J., Borgida, A., Mylopoulos, J., Barone, D., Jiang, L., Yu, E., Amyot, D. Making data meaningful: The business intelligence model and its formal semantics in description logics. In: OTM, pp. 700-717. Springer (2012)

43. Theodorou, Vasileios, Abelló, Alberto, Thiele, Maik, Lehner, Wolfgang. A Framework for User-Centered Declarative ETL. In: DOLAP, pp. 67-70, 2014.

44. Romero, Oscar, Simitsis, Alkis, Abelló, Alberto GEM: Requirement-Driven Generation of ETL and Multidimensional Conceptual Designs. In: Data Warehousing and Knowledge Discovery, pp. 80-95. Springer (2011).

45. Bellatreche, Ladjel, Khouri, Selma, Berkani, Nabila Semantic Data Warehouse Design: From ETL to Deployment á la Carte. In: Database Systems for Advanced Applications, pp. 64-83. Springer (2013).

46. Akkaoui, Zineb, Zimányi, Esteban, Mazón, José-Norberto, Trujillo, Juan. A BPMN-Based Design and Maintenance Framework for ETL Processes. In: IJDWM, 9 (3): 46-72, 2013.

47. Nakuçi, Emona, Theodorou, Vasileios, Jovanovic, Petar, Abelló, Alberto. Bijoux: Data Generator for Evaluating ETL Process Quality. In: DOLAP, pp. 23-32, 2014.

48. Thiele, Maik, Bader, Andreas, Lehner, Wolfgang. Multi-objective scheduling for real-time data warehouses. In: Computer Science - Research and Development, 24 (3): 137-151, 2009.

49. Jovanovic, Petar, Romero, Oscar, Simitsis, Alkis, Abelló, Alberto. Integrating ETL Processes from Information Requirements. In: DaWaK, pp. 65-80, 2012.

50. Theodorou, Vasileios, Abelló, Alberto, Thiele, Maik, Lehner, Wolfgang. POIESIS: a Tool for Quality-aware ETL Process Redesign. In: EDBT, pp. 545-548, 2015.

51. Böhm, Matthias, Wloka, Uwe, Habich, Dirk, Lehner, Wolfgang. GCIP: Exploiting the Generation and Optimization of Integration Processes. In: EDBT, pp. 1128-1131, 2009. 